\title{
Operationalizing a routine wastewater monitoring laboratory for SARS-CoV-2
}

\author{
Rose Kantor ${ }^{1}$, Hannah D. Greenwald ${ }^{1}$, Lauren C. Kennedy ${ }^{1}$, Adrian Hinkle ${ }^{1}$, Sasha \\ Harris-Lovett ${ }^{2}$, Matt Metzger ${ }^{1}$, Melissa M. Thornton ${ }^{1}$, Justin M. Paluba1 ${ }^{1}$, Kara L. Nelson ${ }^{1}$ \\ ${ }^{1}$ Department of Civil and Environmental Engineering, University of California, Berkeley \\ ${ }^{2}$ Berkeley Water Center, University of California, Berkeley
}

\section{Supplementary Text 3}

\section{Supplementary Methods}

The data analysis pipeline was written to convert Cq values to gene copies per well using the slope and intercept from the standard curve on each plate. However, we found that the intercept of the standard curve shifted more than expected from plate to plate (Figure S9). We attribute this to differences in the volume of standard pipetted into the top well of the serial dilution by multiple technicians. To minimize variation in the data due to the standard curve, the data analysis pipeline was used to apply a single average standard curve for SARS-CoV-2 N1 calculated from 145 individual plates. Other sources of plate-to-plate variability were limited by keeping constant the mastermix, plate type, qPCR machine, and primer/probe mixture. To improve consistency of standard curves, a single technician dilutes and aliquots all points on the curve weekly for each assay. This change has substantially improved the consistency of the intercepts across plates. 